\title{
Cuidados Preventivos Para Pneumonia Associada A Ventilação Mecânica: Revisão Integrativa
}

\section{Preventive Care For Pneumonia Mechanical Ventilation Associated: Integrative Review}

\author{
Blenda Gonçalves Cabral' • Aluísio Ferreira Celestino Júnior ${ }^{2} \bullet$ Mary Elizabeth de Santana $^{3}$ \\ Eliseth Costa Oliveira de Matos ${ }^{4}$
}

\begin{abstract}
RESUMO
Introdução: A pneumonia associada a ventilação mecânica é a segunda causa de infecção em pacientes de unidade de terapia intensiva causando altas taxas de mortalidade e de custos hospitalares. $O$ objetivo deste estudo foi identificar na literatura os cuidados mais importantes para prevenir pneumonia associada a ventilação mecânica. Metodologia: Trata-se de estudo exploratório em fontes secundárias, com abordagem qualitativa e utilização do método de revisão integrativa. Resultados: Obteve-se amostragem de 18 artigos publicados, os cuidados considerados mais importantes para prevenção de pneumonia associada a ventilação mecânica adotados pelos profissionais de saúde foram: higiene oral (94,4\%), elevação da cabeceira no leito $30^{\circ}-45^{\circ}(77,7 \%)$, pressão do cuff (66,6\%), aspiração de secreção subglótica $(55,5 \%)$, manutenção do circuito do ventilador $(38,85)$ e higienização das mãos $(33,3 \%)$. Conclusão: O estudo mostra a importância da aplicação destes cuidados para redução na taxa de incidência de pneumonia associada a ventilação mecânica, no tempo de uso do ventilador mecânico e de permanência na unidade de terapia intensiva. E revela que o cuidado menos realizado pelos profissionais é a higienização das mãos considerado um cuidado simples, prático e eficaz para a prevenção de infecções hospitalares.

Palavra-chave: Prevenção de Doenças; Unidades de Terapia Intensiva; Pneumonia Associada à Ventilação Mecânica; Cuidados de Enfermagem
\end{abstract}

\begin{abstract}
Introduction: Pneumonia associated with mechanical ventilation is the second leading cause of infection in intensive care unit patients causing high mortality rates and hospital costs. The objective of this study was to identify in the literature the most important care to prevent pneumonia associated with mechanical ventilation. Methodology: This is an exploratory study in secondary sources, with qualitative approach and use of the integrative review method. Results:A total of 18 published articles were sampled. The most important care for prevention of ventilator-associated pneumonia adopted by health professionals was oral hygiene $(94.4 \%)$, head elevation in bed $30^{\circ}-45^{\circ}(77,7 \%)$, cuff pressure $(66.6 \%)$, aspiration of subglottic secretion (55.5\%), maintenance of the ventilator circuit (38.85) and hand hygiene (33.3\%). Conclusion:The study shows the importance of applying this care to reduce the rate of pneumonia associated with mechanical ventilation, the time of use of the mechanical ventilator and the stay in the intensive care unit. And reveals that the care less carried out by the professionals is the hand hygiene considered a simple, practical and effective care for the prevention of nosocomial infections.

Key-word: Disease Prevention; Intensive Care Units; Pneumonia Associated with Mechanical Ventilation; Nursing Care
\end{abstract}

I. Mestre em Biologia Parasitária na Amazônia pela UEPA, Esp. terapia intensiva em enfermagem. Enfermeira graduada pela Universidade do Estado do Pará.

2. Doutor em Biologia de Agentes Infecciososs e Parasitários, Mestre em Odontologia. Professor Adjunto da Universidade do Estado do Pará e do Centro Universitário do Pará.

3. Profa.Titular da Escola de Enfermagem "Magalhães Barata" da Universidade do Estado do Pará. Doutora em Enfermagem Fundamental pela EERP USP.

4. Doutora em Doenças Tropicais pela UFPA. Prof .Adjunto da Escola de enfermagem "Magalhães Barata” da Universidade do Estado do Pará. 


\section{INTRODUÇÃO}

Pacientes internados em instituições de saúde, particularmente aqueles em Unidade de Terapia Intensiva (UTI), estão frequentemente expostos a uma ampla variedade de microrganismos patogênicos responsáveis por causar infecções graves, devido a sua emergência de resistência bacteriana. Considera-se que este setor representa menos de $2 \%$ dos leitos hospitalares disponíveis, porém contribui com mais de $25 \%$ das infecções hospitalares, com significativo impacto nos índices de morbidade e mortalidade ${ }^{(1-2)}$

Considera-se a UTI um ambiente hospitalar com característica complexa composta de sistema de monitoramento contínuo. Neste local são admitidos pacientes graves, que se apresentam, geralmente, com descompensação por um ou mais sistemas orgânicos. $\mathrm{Na}$ UTI são atendidos pacientes de risco elevado, sendo submetidos ao uso de equipamentos especializados e aos procedimentos invasivos, considerados estes importantes fatores de risco de Infecções Relacionadas a Assistência à Saúde (IRAS), entre eles estão: os cateteres venosos profundos, cateteres arteriais, sondas enterais e vesicais de demora e próteses ventilatórias ${ }^{(3-4)}$

A Pneumonia Associada a Ventilação Mecânica (PAVM) são infeções pulmonares diagnosticadas após $48 / 72$ horas do uso de ventilação mecânica. Considera-se ventilação mecânica uma forma de tratamento artificial utilizado na UTI, com a finalidade de manutenção da oxigenação e/ou ventilação dos pacientes críticos que desenvolvem insuficiência respiratória ${ }^{(5-7)}$

Entre as principais ocorrências de IRAS, verifica-se que a pneumonia se enquadra com $86 \%$ dos casos relacionados ao uso de ventilação mecânica (VM), apresentando alta letalidade, com variação entre $33 \%$ a $71 \%$. Constata-se que a prevalência dos casos fica em torno de 20,5 a 34,4 casos de pneumonia por 1.000 dias de VM e de 3,2 casos por 1.000 dias em pacientes não ventilados $^{(2,4)}$

As taxas de PAVM podem variar conforme a população de pacientes afetada. Mas vários estudos demonstram que a incidência aumenta com a duração da VM e apontam taxas de aproximadamente $3 \%$ por dia durante os primeiros cinco dias de ventilação e depois $2 \%$ para cada dia. A mortalidade varia de 20 a 60\%, mas aproximadamente $33 \%$ dos pacientes com PAVM morrem em decorrência direta da infecção(4)

Ao submeter um paciente à ventilação respiratória, considera-se que $\circ$ paciente apresenta alteração nos seus mecanismos de defesa pulmonar consequência da doença de base, ou ainda pela perda da proteção das vias aéreas superiores. $O$ processo de intubação pode favorecer os distúrbios da fisiologia normal respiratória durante o processo ventilatório, que vai desde a hipersecreção pulmonar até ao aumento da frequência das infecções respiratórias, com elevada taxa de morbidade e de mortalidade ${ }^{(5,8)}$

Dada a importância e a complexidade do problema de saúde, são necessárias as intervenções que causem impactos na prevenção da PAVM, com vistas a atingir uma redução da frequência de IRAS para pneumonia, sendo fundamental o estabelecimento e aplicação das medidas preventivas para estes procedimentos. $\mathrm{Na}$ literatura revelam uma série de recomendações baseadas em evidências que vem com objetivo de melhorar a qualidade da assistência e consequentemente reduzir os custos hospitalares ${ }^{(1,4)}$

A contribuição do estudo foi trazer à atualidade, a discussão sobre a importância de realizar cuidados simples realizados pelos profissionais de saúde apontados na literatura e em órgãos públicos de saúde como eficazes na prevenção e redução da PAVM em todo o processo do cuidar, obedecendo aos procedimentos dentro de conhecimentos técnicos e científicos sobre a temática. Sendo assim o objetivo do estudo foi identificar na literatura os cuidados mais importantes para prevenir a pneumonia associada a ventilação mecânica. Partindo da seguinte questão norteadora: Quais os principais cuidados apontados na literatura sobre prevenção de PAVM?

\section{MÉTODO}

Trata-se de estudo exploratório, com abordagem qualitativa em fontes secundárias, utilizando o método de revisão integrativa.

A Revisão Integrativa da Literatura (RIL) representa apreciável aplicação nos estudos na área da enfermagem. É uma investigação associada à tendência de entender o cuidar em saúde, tanto no âmbito individual como no coletivo, são evidências necessárias na produção de conhecimentos em enfermagem de forma mais ampla ${ }^{(9)}$

A RIL é um método que busca fazer a análise e síntese de vários estudos publicados, tanto experimentais como não experimentais, possibilita um saber fundamentado em conhecimentos científicos e dá uma compreensão mais abrangente dos problemas pertinentes aos cuidados de saúde ${ }^{(10)}$

Dessa forma, recomenda-se esse procedimento quando se deseja realizar uma síntese e análise do conhecimento científico já produzido sobre determinado tema a ser investigado ou ainda quando se pretende adquirir informações que possibilitarão avaliar a pertinência dos procedimentos empregados na elaboração de uma revisão(II)

Para operacionalizar essa revisão, foram seguidas as 
etapas: I) definição do tema e formulação do objetivo e da questão norteadora; 2) estabelecimento dos critérios de inclusão e exclusão dos estudos, 3) busca de dados na literatura, 4) definição das informações a serem extraídas dos estudos selecionados, 5) avaliação dos estudos incluídos, 6) interpretação dos resultados, 7) apresentação da revisão/síntese do conhecimento.

Foram definidos como critério de inclusão: publicações indexadas nas bases de dados e disponíveis on-line, com texto completo, com corte temporal de 2012 a 2016, escritos em português, espanhol e inglês. $E$ considerando como critério de exclusão textos incompletos e artigos que não estivessem disponíveis na íntegra on-line.

As bases de dados pesquisadas para seleção das publicações foram LILACS (Literatura Latino-Americana e do Caribe em Ciências da Saúde), MEDLINE (Literatura Internacional em Ciências da Saúde), BDENF (Base de Dados de Enfermagem), IBECS (Índice Bibliográfico Espanhol de Ciências da Saúde) e CUMED (Biblioteca Virtual em Saúde de Cuba), todas contidas na Biblioteca Virtual em Saúde. Os Descritores em Ciências da Saúde (Decs) foram definidos como "Prevenção","Pneumonia" e "Ventilação Mecânica”.
A busca dos artigos ocorreu com a associação dos descritores: Prevenção AND Pneumonia AND Ventilação Mecânica, obtendo-se como resultados a distribuição de publicações por base de dados. E todo o processo de avaliação e seleção dos artigos são demonstrados na Figura I

Na Prática Baseada em Evidência existe uma classificação de evidência científica caracterizadas de forma hierárquica, dependendo da abordagem metodológica adotada os estudos são classificados em níveis de evidência de $0 \mathrm{I}$ a 06, e os quais são apresentados a seguir, Quadro I.

Para o registro e organização das informações dos artigos selecionados para a revisão, foi elaborado um instrumento de coleta de dados, estruturado em duas categorias nomeadas de: I) dados de identificação dos artigos (título do artigo, título do periódico, autores, país de publicação, idioma, ano de publicação, nível de evidência científica, base de dados) e 2) caracterização metodológica do artigo (objetivo do estudo, tipo de estudo, amostra, local, período de estudo, resultados do estudo, análise dos resultados, limitações e conclusões).

Os dados dos artigos selecionados foram organizados

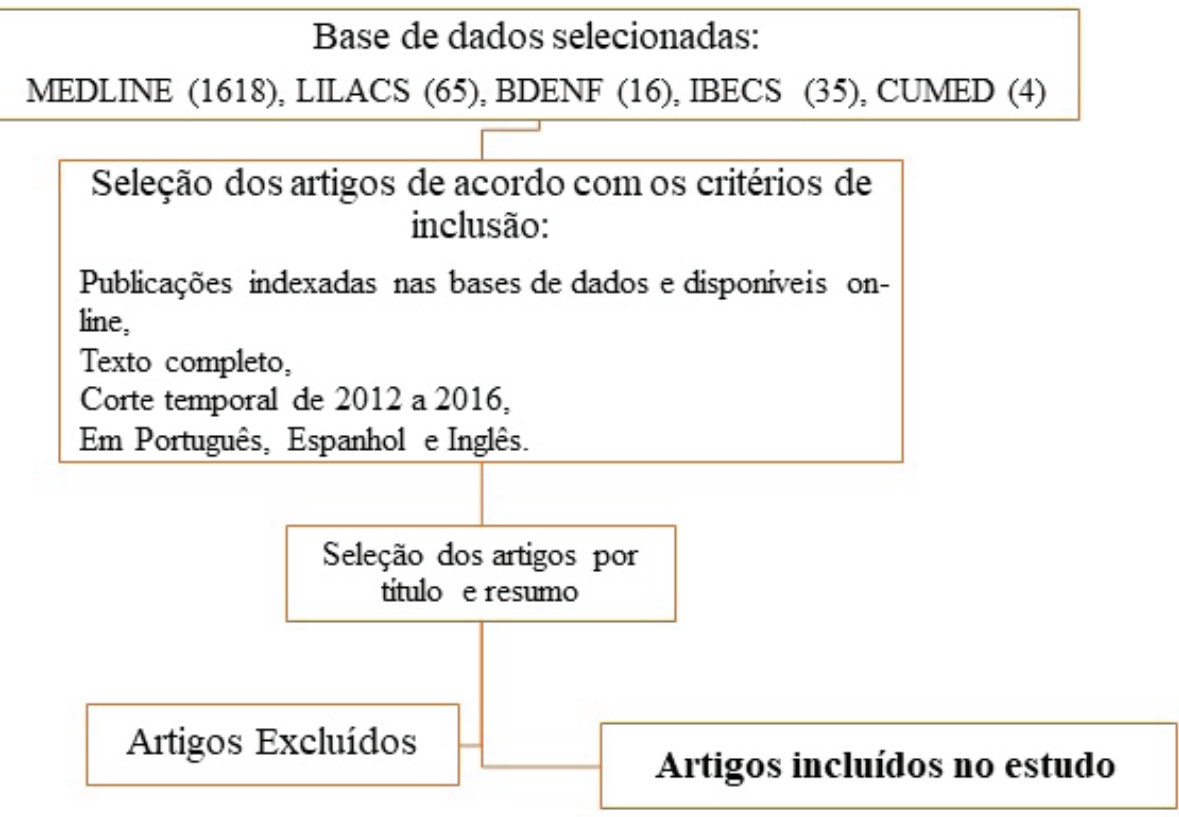

FIGURA 1 - Critérios de busca dos artigos científicos em bases de dados para inclusão na RIL, Belém, PA, Brasil, no período de 2012 a 2016.

QUADRO 1 - Classificação de evidência cientifica segundo (12)

\begin{tabular}{|l|l|}
\hline Nível 01 & Evidências resultantes de meta-analise de múltiplos estudos clínicos controlados e randomizados. \\
\hline Nível 02: & Evidências obtidas em estudos individuais com delineamento experimental. \\
\hline Nível 03: & Evidências de estudos quase-experimentais. \\
\hline Nível 04: & Evidências de estudos descritivos (não experimentais ou com abordagem qualitativa. \\
\hline Nível 05: & Evidencias provenientes de relatos de caso ou de experiência. \\
\hline Nível 06: & Evidencias baseadas em opiniões de especialistas. \\
\hline
\end{tabular}


em uma planilha Microsoft Excel, versão 20I3, e reunidos em tabela a fim de possibilitar a categorização e análise dos mesmos.

As análises realizadas consensualmente, foram aceitas automaticamente. Já as análises realizadas de maneira discordante foram discutidas caso a caso até que se chegasse a um comum acordo entre os pesquisadores. Os dados foram analisados por meio de estatística descritiva e apresentados em quadros e gráficos.

\section{RESULTADOS}

Os dezoito artigos selecionados foram encontrados nas bases de dados LILACS (oito artigos), na MEDLINE (seis), na BDENF (dois) e na IBECS (dois). Estes estudos foram codificados pela letra $A$ (Artigo) seguidos por números (de I a I8), de acordo com a ordem alfabética dos títulos dos artigos, para melhor organização, apresentação e análise dos dados.

De acordo com a classificação de evidência científica, os 18 artigos foram submetidos a análise e classificados em níveis de evidência, como segue: Nível 0 I - (A4 e A I7); Nível 02- (AI0); Nível 03- (A2,A7,AI2,AI8); Nível 04 -
(AI,A3,A5,A6,A8,A9,AII,AI3,AI4,AI5,AI6). Não houve artigos classificados no nível 05 e 06, Quadro 2.

Obteve-se 04 artigos - (AI, A4, A7, AI3) publicados na Espanha; 12 artigos - (A2, A3, A5, A6, A8, A9, AI0, $A|2, A| 4, A I 5, A \mid 6, A I 8)$ no Brasil; 0 lartigo - (AII) em Taiwan; e 0I - (AI7) no Canadá.

Quanto a classificação dos artigos em relação ao idioma e ao ano de publicação foram obtidos para o idioma em inglês seis artigos sendo estes distribuídos respectivamente entre os anos de 2012 com três artigos (AI,A2 e A7), $2013 \mathrm{com}$ dois artigos (AII eA I8) e em $2014 \mathrm{com}$ apenas um artigo (AI7). Já no idioma português houve 10 artigos distribuídos respectivamente no ano de 2012 quatro deles (A3, AIO,AI2 e AI4), do ano de $2013 \mathrm{com}$ um artigo (A8), do ano de 2014 foram três artigos (A5, A9, AI6) e em 2015 foram obtidos dois artigos (A6 e AI5). Para o idioma espanhol foram obtidos dois artigos sendo A4 do ano de 2014 eAI 3 do ano de 20I5, Figura 2.

Quanto aos dados de caracterização metodológica dos artigos, foram analisados os seguintes itens: título; periódico, tipo de estudo; população, resultados - cuidados de prevenção de PAVM (Quadro 3).

\section{QUADRO 2 - Demonstração dos artigos de acordo com a classificação do nível de evidência científica, Belém, PA, Brasil, 2016}

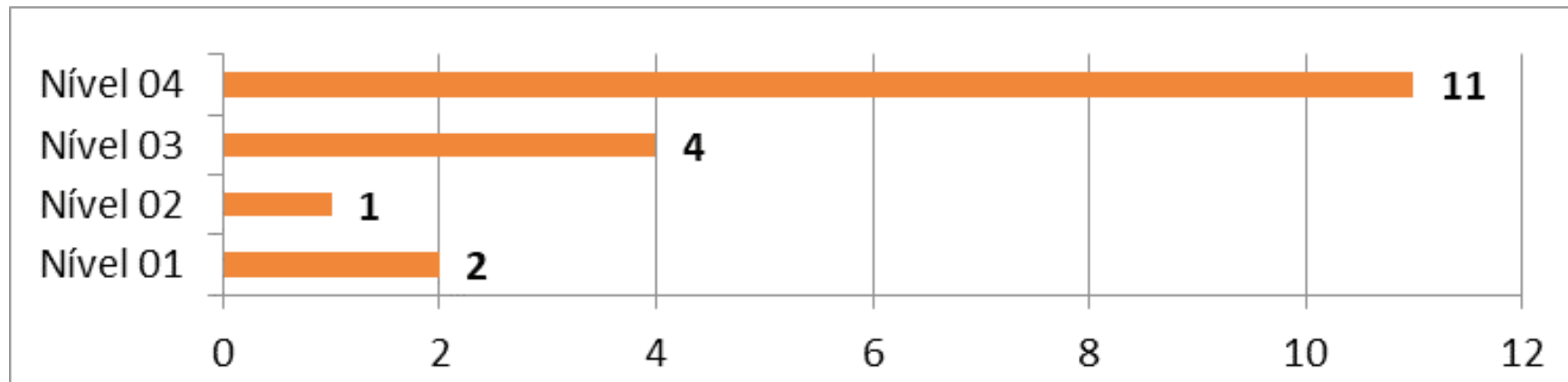

Quantidade de artigos

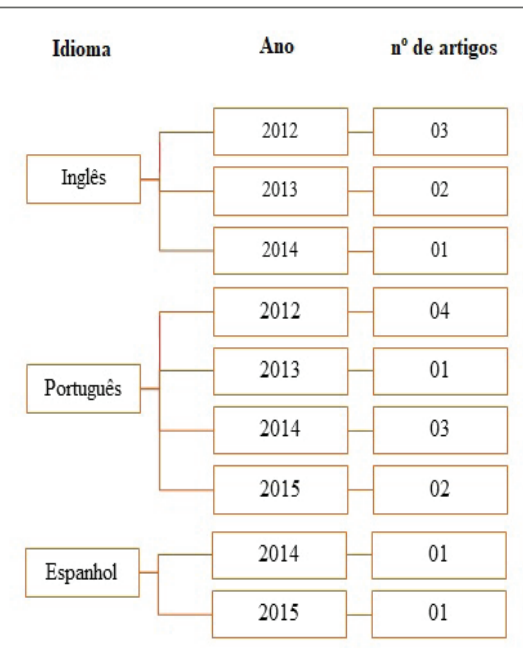

FIGURA 2 - Classificação dos artigos por idioma e ano de publicação, Belém, PA, Brasil, 2016 
QUADRO 3 - Distribuição dos dados obtidos nas publicações após análise dos artigos selecionados pela RIL, Belém, PA, Brasil, 2016

\begin{tabular}{|c|c|c|c|c|c|c|}
\hline ART & Al & A2 & $\mathbf{A 3}$ & A4 & A5 & A6 \\
\hline Título & $\begin{array}{c}\text { A care bundle } \\
\text { approach for } \\
\text { prevention of } \\
\text { ventilator-associated } \\
\text { pneumonia }\end{array}$ & $\begin{array}{l}\text { A program for } \\
\text { sustained improvement } \\
\text { in preventing } \\
\text { ventilator associated } \\
\text { pneumonia in na } \\
\text { intensive care setting }\end{array}$ & $\begin{array}{l}\text { Nursing actions for the } \\
\text { prevention of } \\
\text { ventilator associated } \\
\text { pneumonia }\end{array}$ & $\begin{array}{c}\text { Acciones de } \\
\text { enfermería para la } \\
\text { prevención de la } \\
\text { neumonía associada a } \\
\text { ventilación mecânica: } \\
\text { revisión sistemática }\end{array}$ & $\begin{array}{l}\text { Adesão ás medidas de } \\
\text { um bundle para } \\
\text { prevenção de } \\
\text { pneumonia associada à } \\
\text { ventilação mecânica }\end{array}$ & $\begin{array}{l}\text { Adesão às medidas de } \\
\text { prevenção para } \\
\text { pneumonia associada à } \\
\text { ventilação mecânica }\end{array}$ \\
\hline Periódico & Clin. Microbiol. Infect & Biomed. Central & Acta. Paul. Enferm. & Enfermaria global & $\begin{array}{l}\text { Rev. Bras. Ter. } \\
\text { Intensiva }\end{array}$ & Rev. Enferm. UFSM \\
\hline Tipo de estudo & $\begin{array}{l}\text { Estudo coorte } \\
\text { multicêntrico }\end{array}$ & $\begin{array}{c}\text { Estudo de } \\
\text { delineamento quase- } \\
\text { experimental }\end{array}$ & $\begin{array}{l}\text { Transversal e } \\
\text { observacional }\end{array}$ & $\begin{array}{l}\text { Revisão sistemática } \\
\text { (ensaio clínico- } \\
\text { randomizado) }\end{array}$ & $\begin{array}{c}\text { Descritivo e corte } \\
\text { transversal }\end{array}$ & $\begin{array}{c}\text { Descritivo e } \\
\text { observacional }\end{array}$ \\
\hline População & 1034 pacientes & 21.984 pacientes & 35 profissionais & 13 artigos & & 130 pacientes \\
\hline Resultados & $\begin{array}{c}\text { Redução na } \\
\text { permanência em UTI } \\
\text { no tempo de VM e na } \\
\text { incidência de PAVM }\end{array}$ & $\begin{array}{c}\text { O bundle gerou } \\
\text { redução da } \\
\text { permanência em UTI, } \\
\text { no tempo de VM e na } \\
\text { incidência de PAVM }\end{array}$ & $\begin{array}{l}\text { Os profissionais não } \\
\text { realizaram os cuidados } \\
\text { corretamente }\end{array}$ & $\begin{array}{l}\text { Redução na incidência } \\
\text { de PAVM e dois } \\
\text { cuidados foram } \\
\text { frequentes: } \mathrm{F} \text { e A. }\end{array}$ & $\begin{array}{c}\text { Um bundle obteve } \\
\text { adesão de } 4 \text { cuidados: } \\
\text { A, B, F, O } \\
\text { Pelos profissionais }\end{array}$ & $\begin{array}{c}03 \text { cuidados } \\
\text { demonstraram boa } \\
\text { adesão pelos } \\
\text { profissionais }\end{array}$ \\
\hline Cuidados & $\mathrm{B}, \mathrm{D}, \mathrm{E}, \mathrm{F}, \mathrm{G}$ & $\mathrm{A}, \mathrm{F}, \mathrm{I}, \mathrm{J}, \mathrm{K}$ & $\mathrm{A}, \mathrm{D}, \mathrm{F}, \mathrm{H}, \mathrm{L}$ & $A, C, D, F, H$ & $\mathrm{~A}, \mathrm{~B}, \mathrm{~F}, \mathrm{~N}, \mathrm{O}, \mathrm{P}$ & $A, B, F, G, I, J$ \\
\hline ART & A7 & A8 & A9 & $\mathrm{Al0}$ & All & Al2 \\
\hline Título & $\begin{array}{l}\text { Assessment of a } \\
\text { training programme } \\
\text { for the prevention of } \\
\text { VAP. }\end{array}$ & $\begin{array}{l}\text { Avaliação da } \\
\text { implementação de } \\
\text { novo protocolo de } \\
\text { higiene bucal em um } \\
\text { centro de terapia } \\
\text { intensiva para } \\
\text { prevenção de PAVM. }\end{array}$ & $\begin{array}{l}\text { Evaluation of a bundle } \\
\text { to prevent ventilator- } \\
\text { associated pneumonia } \\
\text { in an ICU }\end{array}$ & $\begin{array}{l}\text { Bundle de prevenção } \\
\text { da PAV: uma } \\
\text { construção coletiva }\end{array}$ & $\begin{array}{l}\text { Efficacy of ventilator- } \\
\text { associated pneumonia } \\
\text { care bundle for } \\
\text { prevention of VAP in } \\
\text { the surgical ICU of a } \\
\text { medical center }\end{array}$ & $\begin{array}{l}\text { Eficácia de estratégias } \\
\text { educativas para ações } \\
\text { preventivas da PAVM }\end{array}$ \\
\hline Periódico & Nurs. in Critical & Rev. Min. Enferm. & $\begin{array}{c}\text { Texto \& Contexto } \\
\text { Enferm. }\end{array}$ & $\begin{array}{c}\text { Texto \& Contexto } \\
\text { Enferm. }\end{array}$ & \begin{tabular}{|c}
$\begin{array}{c}\text { J. Microbiol. Immunol. } \\
\text { Infect. }\end{array}$ \\
\end{tabular} & $\begin{array}{l}\text { Esc. Anna Nery Rev. } \\
\text { Enferm. }\end{array}$ \\
\hline Tipo de estudo & Prospectivo & $\begin{array}{c}\text { Descritivo e } \\
\text { observacional }\end{array}$ & $\begin{array}{c}\text { Descritivo e } \\
\text { transversal }\end{array}$ & $\begin{array}{l}\text { Covergente- } \\
\text { assistencial }\end{array}$ & Estudo retrospectivo & $\begin{array}{l}\text { Ensaio clínico não- } \\
\text { randomizado }\end{array}$ \\
\hline População & 58 enfermeiros & 56 profissionais & 81 profissionais & 25 profissionais & 28.454 pacientes & 35 profissionais \\
\hline Resultados & $\begin{array}{l}\text { Cuidados com boa } \\
\text { adesão foram: } \mathrm{B}, \mathrm{C}, \mathrm{F} \text {, } \\
\mathrm{W} \text {. }\end{array}$ & $\begin{array}{c}\text { Reduziu a taxa de } \\
\text { incidência de PAVM } \\
\text { com a implantação do } \\
\text { Bundle }\end{array}$ & $\begin{array}{c}\text { Os cuidados } \mathrm{C} \text { e F } \\
\text { alcançaram } \geq 80 \% \text { de } \\
\text { conformidade }\end{array}$ & $\begin{array}{c}\text { Os profissionais } \\
\text { colaboraram para a } \\
\text { construção do Bundle }\end{array}$ & $\begin{array}{l}\text { Redução da taxa de } \\
\text { PAVM, no uso de } \\
\text { VM. E adesão dos } \\
\text { cuidados em >95\% }\end{array}$ & $\begin{array}{l}\text { Os profissionais } \\
\text { realizaram os } \\
\text { cuidados: F, D e H } \\
\text { com eficácia }\end{array}$ \\
\hline Cuidados & $A, B, F, W$ & $\begin{array}{c}\text { A, B, C, D, E, F, G, H, } \\
\text { I, J, L, O }\end{array}$ & $A, B, C, F$ & $\mathrm{~A}, \mathrm{~B}, \mathrm{C}, \mathrm{F}$ & $\mathrm{A}, \mathrm{B}, \mathrm{C}, \mathrm{D}, \mathrm{F}$ & $\begin{array}{c}\mathrm{F}, \mathrm{B}, \mathrm{D}, \mathrm{A}, \mathrm{H}, \mathrm{C}, \mathrm{Q}, \mathrm{R} \text {, } \\
\mathrm{S}, \mathrm{T}\end{array}$ \\
\hline ART & Al3 & Al4 & Al5 & Al6 & Al7 & A18 \\
\hline Titulo & $\begin{array}{c}\text { Evaluación del } \\
\text { cumplimiento de um } \\
\text { protocolo de } \\
\text { prevención de } \\
\text { neumonía associada a } \\
\text { ventilación mecánica } \\
\text { en una UCI polivalente }\end{array}$ & $\begin{array}{l}\text { Impacto da aspiração } \\
\text { supra-cuff na } \\
\text { prevenção da } \\
\text { pneumonia associada a } \\
\text { ventilação mecânica }\end{array}$ & $\begin{array}{l}\text { Pneumonia associada à } \\
\text { ventilação mecânica } \\
\text { como indicador de } \\
\text { qualidade e segurança } \\
\text { em saúde }\end{array}$ & $\begin{array}{l}\text { Pneumonia associada à } \\
\text { ventilação mecânica: } \\
\text { discursos de } \\
\text { profissionais acerca da } \\
\text { prevenção }\end{array}$ & $\begin{array}{c}\text { Prophylactic oral } \\
\text { health procedures to } \\
\text { prevent hospital } \\
\text { acquired and ventilator } \\
\text { associated pneumonia: } \\
\text { a systematic review }\end{array}$ & $\begin{array}{c}\text { Ventilator- associated } \\
\text { pneumonia prevention } \\
\text { by education and two } \\
\text { combined bedside } \\
\text { strategies }\end{array}$ \\
\hline Periódico & Enfermeria global & $\begin{array}{l}\text { Rev. bras. Ter. } \\
\text { intensiva }\end{array}$ & $\begin{array}{l}\text { Rev. Med. Minas. } \\
\text { Gerais }\end{array}$ & $\begin{array}{l}\text { Esc. Anna Nery Rev. } \\
\text { enferm. }\end{array}$ & Int. J. Nurs. Stud. & $\begin{array}{l}\text { Int. J. Qual. Health } \\
\text { Care }\end{array}$ \\
\hline Tipo de estudo & $\begin{array}{l}\text { Descritivo e } \\
\text { retrospectivo }\end{array}$ & Revisão de literatura & $\begin{array}{c}\text { Retrospectivo, } \\
\text { descritivo e } \\
\text { comparativo } \\
\end{array}$ & $\begin{array}{c}\text { Descritiva abordagem } \\
\text { qualitativa }\end{array}$ & $\begin{array}{c}\text { Revisão sistemática } \\
\text { ensaio clínico } \\
\text { randomizado } \\
\end{array}$ & $\begin{array}{c}\text { Ensaio clínico não } \\
\text { controlado }\end{array}$ \\
\hline População & 94 pacientes & - & - & 25 profissionais & 28 artigos & 518 \\
\hline Resultados & $\begin{array}{l}\text { Redução nas taxas de } \\
\text { PAVM e dois cuidados } \\
\text { não foram aderidos } \\
\text { pelos profissionais }\end{array}$ & $\begin{array}{l}\text { Redução na incidência } \\
\text { de PAVM, no uso de } \\
\text { VM e permanência em } \\
\text { UTI }\end{array}$ & $\begin{array}{c}\text { Com o bundle houve } \\
\text { redução na incidência } \\
\text { de PAVM e no tempo } \\
\text { de uso de VM }\end{array}$ & $\begin{array}{c}\text { Os profissionais } \\
\text { mostraram } \\
\text { conhecimento sobre } \\
\text { prevenção de PAVM }\end{array}$ & \begin{tabular}{|c|} 
Redução na incidência \\
de PAVM e na \\
permanência no \\
hospital após a \\
realização dos \\
cuidados \\
\end{tabular} & $\begin{array}{c}\text { Houve redução na } \\
\text { incidência de PAVM } \\
\text { com as intervenções } \\
\text { educativas e } \\
\text { implantação do bundle }\end{array}$ \\
\hline Cuidados & $\mathrm{E}, \mathrm{A}, \mathrm{F}, \mathrm{B}, \mathrm{C}, \mathrm{M}$ & $\mathrm{C}$ & $A, F, B, V, I, E$ & $\mathrm{E}, \mathrm{F}, \mathrm{X}, \mathrm{C}, \mathrm{D}, \mathrm{K}, \mathrm{B}, \mathrm{A}$ & $\mathrm{F}$ & $A, C, B, F$ \\
\hline
\end{tabular}

Legenda: Elevação da Cabeceira (A); Pressão-Cuff (B); Aspiração de Secreções Subglóticas (C); Manejo do Circuito do Ventilador (D); Higienização das mãos (E); Higienização Oral (F); Monitorização de sedação (Desmame) (G); Higienização Brônquica (H); Profilaxia de Trombose Venosa (I); Profilaxia de Úlcera Peptídica (J); Avaliação de Extubação (K); Administração de Dieta (L); Utilização de Umidificador (N); Posição do Filtro Umidificador (O); Fisioterapia (P); Troca de Nebulizador (Q); Mudança de Decúbito (R); Uso de antisséptico (S); Uso de EPI (T); Aspiração Gástrica (U); Profilaxia de Úlcera Gástrica (V); Prevenção de Bronco-Aspiração (X); Fixação do Tubo Endotraqueal (W).

Feito a análise dos objetivos de cada estudo, foi obtido como resultado a formação de sete grupos, os quais agruparam artigos que possuíam semelhança nos seus objetivos (Figura 3).

Foram obtidos um total de 24 cuidados propostos nos 18 artigos, dentre estes cuidados houve seis que foram mais frequentes nos 18 artigos, sendo eles: I. Elevação da cabeceira; 2. Pressão-cuff; 3. Aspiração subglótica; 4 . Manejo do circuito do ventila- dor, 5. Higienização das mãos e 6. Higienização oral (Quadro 4).

De acordo com análise feita o cuidado Higienização oral foi o mais frequente, presente em 17 artigos. $O$ cuidado Elevação da Cabeceira 30 - $45^{\circ}$ foi presente em 14 artigos, o cuidado Pressão-Cuff presente em 12 artigos; 0 cuidado Aspiração de secreções subglóticas em 10 artigos; o cuidado Manejo do circuito do ventilador em sete artigos e o cuidado higienização das mãos em seis artigos. 


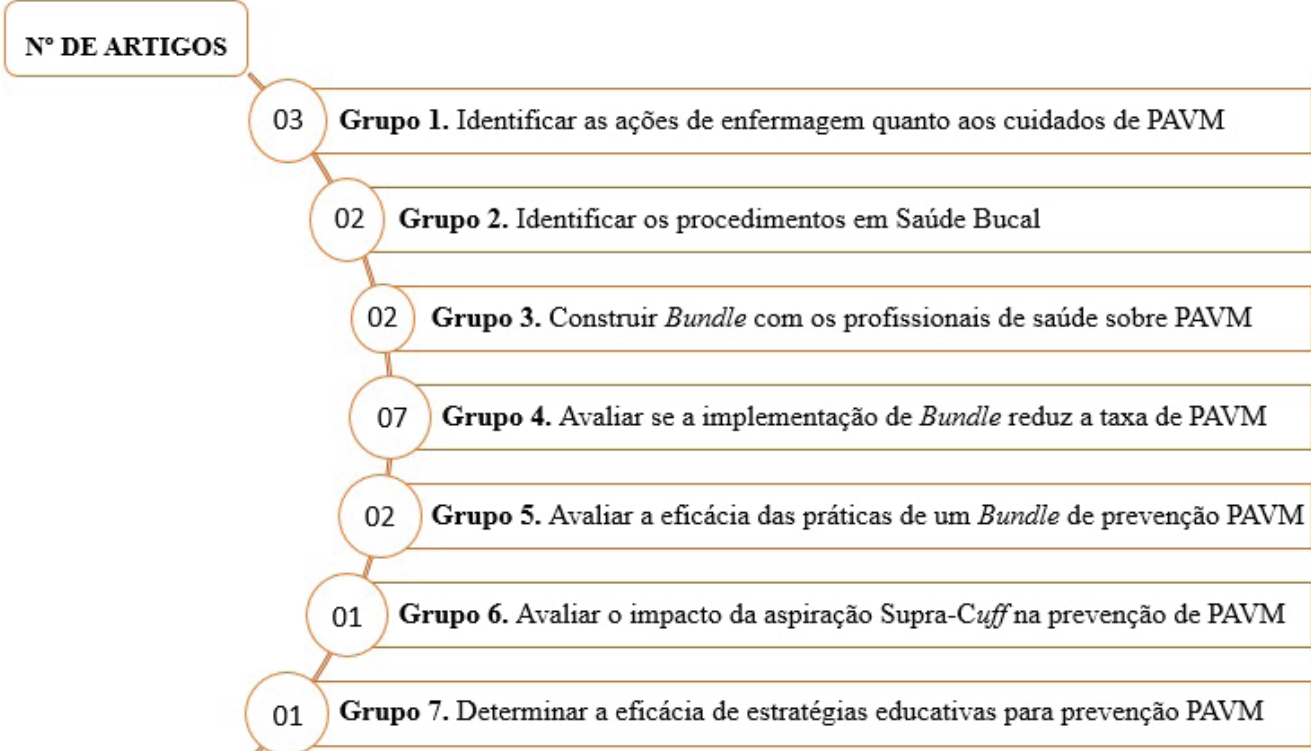

FIGURA 3 - Distribuição dos agrupamentos dos artigos, de acordo com as propostas dos estudos, Belém, PA, Brasil, 2016.

QUADRO 4 - Distribuição dos cuidados para prevenção de PAVM obtidos após análise dos 18 artigos publicados, Belém, PA, Brasil, no período de 2012 a 2016.

\begin{tabular}{|l|r|}
\hline \multicolumn{1}{|c|}{ CUIDADOS } & $\begin{array}{c}\text { Frequência dos } \\
\text { cuidados/ART. }\end{array}$ \\
\hline HIGIENIZAÇÃO ORAL & 17 \\
\hline ELEVAÇÃO DA CABECEIRA & 14 \\
\hline PRESSÃO-CUFF & 12 \\
\hline ASPIRAÇÃO SUBGLÓTICA & 9 \\
\hline MANEJO DO CIRCUITO DO VENTILADOR & 7 \\
\hline HIGIENIZAÇÃO DAS MÃOS & 6 \\
\hline MONITORIZAÇÃO PARA DESMAME & 5 \\
\hline HIGIENIZAÇÃO BRÔNQUICA & 5 \\
\hline PROFILAXIA DE TROMBOSE VENOSA & 3 \\
\hline ADMINISTRAÇÃO DE DIETA & 3 \\
\hline PROFILAXIA DE ÚLCERA PEPTÍDICA & 2 \\
\hline AVALIAÇÃO DE EXTUBAÇÃO & 2 \\
\hline MONITORIZAÇÃO DE LÍQUIDO NO VENTILADOR & 2 \\
\hline USO DE EPI & 2 \\
\hline ASPIRAÇÃO GÁSTRICA & 2 \\
\hline FISIOTERAPIA & 1 \\
\hline TROCA DE NEBULIZADOR & 1 \\
\hline MUDANÇA DE DECÚBITO & 1 \\
\hline USO DE ANTISÉPTICO & 1 \\
\hline POSIÇÃO DO FILTRO UMIDIFICADOR & 1 \\
\hline UTILIZAÇÃO DE UMIDIFICADOR & 1 \\
\hline PROFILAXIA DE ÚLCERA GÁSTRICA & 1 \\
\hline PREVENÇÃO DE BRONCOASPIRAÇÃOO & 1 \\
\hline FIXAÇÃO DE TUBO ENDOTRAQUIAL & 1 \\
\hline
\end{tabular}

\section{DISCUSSÃO}

\section{Higienização Oral}

Os artigos que abordaram o cuidado higienização oral foram AI, A2, A4, A5, A7, A8, A9, AII,AI2,AI3,AI7 e A 18 , estes relatam que houve boa adesão do cuidado pelos profissionais de saúde, além de demonstrar resul- tados positivos quanto a redução na taxa de incidência de PAVM ${ }^{(13)}$

Outro aspecto importante ressaltado sobre este cuidado foi o uso de clorexidina $0,12 \%$ durante o processo de higienização da cavidade oral. Os estudos comprovaram que a utilização dessa substância reduz a presença 
de microrganismos na cavidade oral e também na placa dental no percentual de até $88 \%$ após 5 horas da sua administração, pois ela tem ação bactericida, atuando contra bactérias Gram-negativas e positivas; além disso, é considerada padrão-ouro como antisséptico para pacientes intubados ${ }^{(14)}$

Pacientes que são submetidos ao uso de ventilação mecânica, apresentam em sua grande maioria, alguma doença de base respiratória, o que leva ao comprometimento do processo respiratório relacionado às trocas gasosas de $\mathrm{O}_{2}$ e $\mathrm{CO}_{2}$. Logo o paciente é intubado, a fim de dar suporte nas trocas gasosas, e sedado, pois, o uso do tubo endotraqueal associado ao ventilador mecânico causa um desconforto no trato respiratório, ao ser introduzido. Com estabelecimento deste quadro, os pacientes ficam impossibilitados de mastigar e deglutir ocorrendo então a hipossalivação, favorecendo assim a formação de biofilme dental, considerado um reservatório de patógenos virulentos. Além disso, pacientes com outras comorbidades sofre mais risco a infecção por pneumonia, dentre as comorbidades estão: DPOC, insuficiência cardíaca, diabetes mellitus, principalmente quando internados em UTI. O Institute for Healthcare Improvement ressalta que com a realização da higienização oral o acúmulo de patógenos na cavidade oral ou de biofilme é reduzido, devido a utilização de técnica correta e uso de clorexidina ${ }^{(15)}$

Os pacientes submetidos em ventilação mecânica possuem maior probabilidade de colonização por bactérias virulentas na flora bucal, por esta razão a higienização bucal é indispensável, além disso, a flora bucal desencadeia a colonização de bactérias no pulmão, este mecanismo se dar da seguinte forma: na via bucal há a colonização de bactérias, e com a larga utilização de antibióticos no tratamento dos pacientes, gera-se a baixa produção de saliva formando um biofilme com alta taxa de bactérias virulentas, quando não realizado a higienização oral o paciente acumula secreções espessas (muco com microrganismos), que migram para a região subglótica devido à ausência do mecanismo de deglutição e posteriormente passam para a árvore brônquica levando ao acúmulo de muco no pulmões resultando em infecção.

A higienização oral por ser um cuidado simples, fácil e de baixo custo além de combater as infecções de forma eficaz necessita ser enfatizado nas instituições e imposto como procedimento de rotina além de ser fiscalizado como controle de infecções, principalmente relacionados a controle de infecções pulmonares. Esse mecanismo de infecção pode ser controlado com a realização desse simples cuidado. Evitando não somente a infecção do paciente, mas a preservação das necessidades humanas básicas do paciente de UTI. Portanto é de suma importância que os profissionais de saúde recebam treinamentos e sejam conscientizados da importância do cuidado, já que a higienização da flora bucal reduz em $40 \%$ as pneumonias associadas à ventilação mecânica ${ }^{(16)}$

\section{Elevação da Cabeceira}

A elevação da cabeceira em $30-45^{\circ}$ foi abordado pelos artigos A2, A3, A4, A5, A6, A7, A8, A9, AI0,AII,AI2, AI3, AI5 eA I8.A importância deste cuidado se dá pelo fato de que pacientes intubados e posicionados com a cabeceira elevada em $30-45^{\circ}$ não sofrem broncoaspiração e consequentemente não ocorre PAVM, contribuindo para uma melhoria no volume corrente e ventilatório, e minimizando os casos de atelectasia, embora esta seja uma prática simples e sem custos adicionais, é necessário ser realizada com responsabilidade e valorizada pelos profissionais, visto que, configura um cuidado essencial na prevenção da pneumonia associada ao ventilador mecânico ${ }^{(17)}$

A elevação da cabeceira a $30-45^{\circ}$ é um cuidado simples e de fácil realização, no entanto, pode-se observar na análise dos 18 artigos que alguns deles relataram baixa adesão da elevação da cabeceira pelos profissionais de saúde devido a difícil monitorização deste cuidado com o paciente, já que ao longo do dia, os pacientes são submetidos a vários procedimentos como: banho no leito, mudança de decúbito, administração de medicamentos e dieta, além disso, relataram também que os pacientes tendem a escorregar no lençol quando assumem esta posição na cabeceira ${ }^{(16-17)}$

Como alternativa para sugere-se a adesão pela posição do paciente em trendelenburg lateral, contribui na prevenção de PAVM, visto que, este posicionamento além de evitar aspiração pulmonar contribui para limpeza do muco em pacientes intubados ${ }^{(17)}$

\section{Pressão-Cuff}

$O$ cuidado Pressão-cuff do tubo endotraqueal foi apresentado pelos artigos AI,A5,A6,A7,A8,A9,AI0,AII, $A|2, A| 3, A \mid 5$ e $A \mid 8$, e consiste em monitorizar a pressão do balonete de 4 em $4 \mathrm{~h}$ fazendo uso do cuffômetro (instrumento medidor de pressão do cuff, a qual deve estar entre 20 a $30 \mathrm{~cm} \mathrm{H2O}$, valor recomendado pela Diretriz de pneumonia associada a ventilação mecânica. Este cuidado está diretamente relacionado a prevenção de PAVM, pois a monitorização da pressão do cuff possibilita a vedação da traquéia ao ponto de prevenir broncoaspirações, definida como a aspiração de conteúdo gástrico ou corpo estranho na árvore traqueobrônquica que pode levar a obstrução das vias aéreas e de evitar o comprometimento da perfusão traqueal. No entanto, se ocorrer uma hiperinsuflação do cuff, ou seja, pressões acima de $30 \mathrm{~cm} \mathrm{H}_{2} \mathrm{O}$ é provável que aconteça danos aos pacientes como: isquemia da mucosa, lesão de cartilagem, estenose e fístula traqueoesofágica ${ }^{(18-19)}$

De acordo com a análise dos artigos citados ante- 
riormente, a pressão-cuff não registrou boa adesão pelos profissionais, e verificou-se que a pressão-cuff estava entre 10 a $30 \mathrm{~cm} \mathrm{H}_{2} \mathrm{O}$, abaixo da recomendada. E pressões do cuff abaixo do recomendado ocasionam broncoaspiração, elevando o risco de aspiração de secreções acumuladas acima do cuff, provenientes da região orofaríngea contaminada com conteúdo gástrico ou secreções orais colonizadas por bactérias que contribuem para a ocorrência de infecções pulmonares, destacando-se entre elas a PAVM.A monitorização da pressão do cuff deve ser um cuidado padronizado e adotado pela equipe atuante em UTI, tendo trocas a cada 8- 12 horas por turno devido à instabilidade do cuff de manter sua pressão interna por períodos prolongados ${ }^{(19)}$

\section{Aspiração de secreções subglóticas}

Os artigos que ressaltaram o cuidado de aspiração subglótica foram $A 4, A 7, A 8, A 9, A|0, A| I, A|2, A| 3$, Al4, Al6.

A aspiração de secreções subglóticas trata-se de um cuidado indispensável em pacientes em ventilação mecânica, pois a presença de uma via artificial (tubo endotraqueal) interfere no reflexo de tosse, devido a perda da barreira natural entre a orofaringe e a traqueia, culminando no acúmulo de secreções acima do cuff, proporcionando maior colonização da árvore traqueobrônquica e predispondo a migração de secreções para as vias aéreas inferiores, prejudicando a ventilação. Portanto, a aspiração de secreções subglóticas tem $\circ$ intuito de diminuir o acúmulo dessas secreções e manter as vias aéreas desobstruídas e reduzindo o risco da consolidação de atelectasia, que podem ser ocasionadas de uma ventilação inadequada ${ }^{(20)}$

A remoção de secreções é indispensável, no entanto, requer avaliação criteriosa antes de ser realizada, é recomendado que seja feito a ausculta pulmonar a fim de certificar se há necessidade de realizar a aspiração de secreções, pois a realização desse cuidado em períodos curtos (de hora em hora) pode desencadear em hipoxemia, instabilidade cardiovascular, aumento da pressão intracraniana, lesões da mucosa e infecções ${ }^{(21)}$

Nos artigos que aderiram a aspiração de secreção subglótica, foi observado que este cuidado teve boa adesão pelos profissionais de saúde e a sua realização reduziu a incidência de PAVM, além de diminuir o tempo de uso de ventilação mecânica. O Al4 destacou uma avaliação entre aspiração de secreção por sistema fechado e aberto e comprovou que o sistema fechado é mais eficaz, pois neste sistema não há desconexão do tubo endotraqueal com o ventilador mecânico, possibilitando que o processo de aspiração ocorra no interior do tubo livre de contaminação e infecções por parte do manuseio direto do aparelho pelos profissionais de saúde. No entanto, não houve diferença entre os sistemas quanto a redução na taxa de incidência de PAVM ${ }^{(22)}$

A adoção de uma técnica asséptica baseada em conhecimento científico quando realizada, minimiza os riscos de colonização dos microrganismos pelas vias aéreas inferiores do paciente prevenindo a PAVM.

\section{Manejo do circuito do ventilador}

O cuidado manejo do circuito do ventilador foi mencionado pelos artigos AI, A3, A4, A8, AII, AI2 e AI6. É importante que os profissionais tenham conhecimento a respeito de como este equipamento deve ser montado e manuseado. É recomendado que a traquéia esteja livre de água condensada e que o manejo no circuito do ventilador, ocorra apenas em caso de sujidade, ou falha no funcionamento do aparelho, porém a verificação do bom funcionamento do aparelho deve ser feita durante todo o período de intubação do paciente.

Os artigos constataram que os profissionais de saúde precisam de treinamento a respeito do manejo do circuito do ventilador, em relação a montagem do aparelho ${ }^{(22-23)}$

Outros cuidados necessários com o ventilador mecânico é a higienização do Tubo orotraqueal (TOT) diariamente, a fim de evitar o acúmulo de secreções no interior do tubo e consequentemente a colonização por microrganismos multirresistentes, na grande maioria dos casos de pacientes internados em UTI.

É importante destacar que a higienização do tubo deve ser realizada com cuidado para não perfuração do mesmo, associado a este processo está o umidificador, o qual deve ser preenchido corretamente com filtros que funcionam como barreira para a entrada de microrganismos. Porém em pacientes hipersecretivos o procedimento anteriormente citado é contra indicado, pois os poros dos filtros podem ser obstruídos pela secreção do paciente durante o processo de filtração.

Durante a troca do fixador do tubo é recomendado que se utilize um equipo de auxilio, evitando a desconexão de um dos ramos do circuito. Portanto, o cuidado com o circuito do ventilador mecânico para promoção de uma adequada ventilação pulmonar e consequente prevenção da PAVM mostrou-se um dos cuidados mais importantes. Porém, o manuseio do ventilador foi o cuidado com menor frequência de realização entre os profissionais de saúde, sendo necessário a realizações de treinamentos e conhecimento científico relacionado a este cuidado para os profissionais de saúde.

\section{Higienização das mãos}

E o cuidado higienização das mãos foi o menos abordado pelos artigos analisados, mencionado apenas pelos artigos $\mathrm{Al}$, A8, AII, AI 3, AI5 e AI6. Foi observado tam- 
bém que o cuidado da higienização das mãos foi pouco realizado pelos profissionais de saúde ${ }^{(23)}$

Este dado é preocupante já que a higienização das mãos é recomendada como medida eficaz para a prevenção de infecções em geral e no controle de transmissão das infecções, por ser uma medida que reduz a microbiota residente nas mãos dos profissionais interrompendo a cadeia de transmissão da doença. Essa prática associada ao uso de equipamentos de proteção individual (EPI) contribui para prevenção e controle de infecções, incluindo a PAVM, que é uma das infecções mais frequentes nas UTI. E, além disso, este cuidado é de simples realização e de baixo custo. E um importante cuidado na prevenção de $\operatorname{PAVM}^{(4,8,23)}$

O cuidado de higienização das mãos deveria ser 0 cuidado mais realizado, pela facilidade da técnica, o baixo custo e a importância do mesmo na prevenção e controle de infecções, no entanto, foi o cuidado menos realizado pelos profissionais de saúde e quando realizado, a técnica era inadequada ou incorreta, demonstrando a falta de interesse dos profissionais quanto a higienização das mãos assim como, a falta de adesão deste cuidado antes e depois dos procedimentos.

A não adesão do cuidado de higienização das mãos pelos profissionais é um dos principais fatores para o desenvolvimento de infecções principalmente a PAVM.

Os artigos também apontaram um resultado importante sobre a realização de ações educacionais continuadas com os profissionais de saúde, pois constataram que quando os Bundles eram implementados junto com uma ação educacional, treinamento ou outras tecnologias educativas, a adesão aos cuidados de prevenção de PAVM e a obtenção e esclarecimento de informações eram mais satisfatórias. Além de enfatizarem que a permanência de educação continuada com os profissionais de saúde e a prática correta dos cuidados para prevenção de PAVM reduzem as taxas de incidência de PAVM, nas UTI ${ }^{(14,22)}$

\section{CONCLUSÃO}

Com este estudo foi possível evidenciar que a capacitação contínua dos profissionais de saúde é fundamental para garantir uma assistência com qualidade e segurança ao paciente. Os achados da pesquisa em análise revelam que quando a equipe aplica as medidas preventivas em PAVM há redução do risco de adquirir infecção no trato respiratório e ocorre redução do tempo de internação do paciente, reduzindo consequentemente a taxa de mortalidade por esta patologia nas UTI. 


\section{REFERÊNCIAS}

I. Gomes AC, Carvalho PO, Lima ETA, Gomes ET,Valença MP, Cavalcanti ATA. Characterization of infections related to health care in the intensive care unit. Rev Enferm UFPE on line. 20 | 4; 8 (6): I 577-85.

2. SANTOS, BSP. DOS; RIBEIRO, IP; CAMPELO, SMA; CARVALHO, HEF DE; SOUSA, BS DE A; VALLE, ARM DA C. Compreensão do Familiar Acompanhante sobre Prevenção das Infecções Relacionadas à Assistência em Saúde. Revista Enfermagem Atual InDerme, v. 86, n. 24, 10 dez. 2018.

3. Bork LCA, Gaspar MDR, Reche PM. Adesão às medidas preventivas de pneumonia associada à ventilação mecânica. Rev Epidemiol Control Infect [Internet]. 2015 [acesso em 17 jul 2018]; 5 (1):I2-I6. Disponível em: http://dx.doi. org/I0.17058/reci.v5il.4885

4. Manfredini CS, Marco SL, Denti IA. Prevalência de infecções em uma Unidade de Terapia pediátrica e neonatal. Rev Perspectiva. 20I4; 38 (I):73-8I.

5. Oliveira MLL, Rodrigo DN. Bundles de prevenção da pneumonia associada à ventilação mecânica em unidade de terapia intensiva. Rev Amazônia Science \& Health. 2015; 3 (2):36-43.

6. Arens C, Bajwa SA, Koch C, Siegler BH, Schneck E, Hecker A, Weiterer S, Lichtenstern C, Weigand MA, Uhle F. Sepsis -induced long-term immune paralysis-results of a descriptive, explorative study. Crit Care [Internet]. 2016 [acesso em 20 jul 2018]; 20:93. Disponível em: https://doi.org//0.1 I86/ sl3054-0|6-1233-5

7. Choudhuri AH.Ventilator-Associated Pneumonia:When to hold the breath? Int J Crit IIIn Inj Sci [Internet]. 2013 [acesso em 10 set 20I6]; 3 (3):I69-74. Disponível em: https:// doi.org/I0.4103/2229-5151.119195

8. Perugini MRE, Perugini VH, Figueira FD, Fontana LMS, Diniz JJ, Santos DL, Belei RA, Vespero EC, Pelisson M, Stipp-Abe AT, Capobiango JD. Impacto de um Bundle nas taxas de pneumonia associada à ventilação mecânica (PAV) em uma unidade de terapia intensiva pediátrica em Londrina-PR. Semina Cienc Biol Saude. 20I5; 36 (I):259-266.

9. Nepomuceno RM, Miranda CB, Nogueira C, Silva LCF, Silva LD. Modifiable risk factors for mechanical ventilator associated pneumonia in intensive care. Rev Epidemiol Control Infect. 20I4; I6 (I):23-27.

10. Soares CB, Hoga LAK, Peduzzi M, Sangaleti C, Yonekura T, Silva DRAD. Integrative Review: Concepts and methods used in Nursing. Rev esc Enferm USP. 20I4; 48 (2):335-45.

II. Santos GAC, Silva JR, Matos ECO, Santana ME. Dengue: Prevenção, Controle e Cuidados de Enfermagem - Revisão Integrativa da Literatura 2008-20 I3. Rev Bras Cienc Saude. 2016; 20 (I):7I-78.

12. Botelho LLR, Cunha CCA, Macedo M. Método da revisão integrativa nos estudos organizacionais. Gestão e Sociedade [Internet]. 20II [acesso em 26 mai 20I6]; 5 (I I): I2I-
136. Disponível em: https:/www.gestaoesociedade.org/ gestaoesociedade/article/view//220/906

13. Souza MT, Silva MD, Carvalho R. Revisão integrativa: o que é e como fazer? Einstein [Internet]. 2010 [acesso em 06 nov 2016]; I(8):I02-106. Disponível em: http://dx.doi. org/I0.1590/s I679-450820 I0rwII34

14. Silva AAL, Gazzineli A. Knowledge of nursing professionals in relation to measures of prevention of infections. Rev Enferm UFPE on line. 2017; I I (I): 18-23

I5. Elbaky MMA, Youssef IA, Soussi AHE. Improving Internship Knowledge and Performance about Oral Hygiene Bundle for Mechanically Ventilated Patients. J Nurs Health Sci. 2015; 04 (4): 53-59.

16. Institute for Healthcare Improvement. 5 million lives campaign. getting started kit: prevent ventilator associated pneumonia how-to guide. Cambridge, MA (US): Institute for Healthcare Improvement; 2014.

17. Martinez BP, Marques TI, Santos, DR, Silva VS, Nepomuceno BR, Alves GAA, Gomes M, Forgiarini LA. Influência de diferentes graus de elevação da cabeceira na mecânica respiratória de pacientes ventilados mecanicamente. Rev Bras Ter Intensiva. 20I5; 27 (4):347-352.

18. Annoni R, Pires-Neto RC. Ineffectiveness of using the pressure relief valve technique during cuff inflation. Rev Bras Ter Intensiva. 20।4; 26 (4):367-372

19. Gonçalves RL, Tsuzuki LM, Carvalho MGS. Endotracheal suctioning in newborns:in integrative literature review. Rev Bras Ter Intensiva. 201 5; 27 (3):284-292.

20. Damas P, Frippiat F, Ancion A, Canivet JL, Lambermont B, Layios N, Massion P, Morimont P, Nys M, Piret S, Lancellotti P, Wiesen P, D'orio V, Samalea N, Ledoux D. Prevention of ventilator-associated pneumonia and ventilator-associated conditions: a randomized controlled trial with subglottic secretion suctioning. Crit Care Med. 20I5; 43 (I):22-30.

21. Chicayban LM, Terra ELVS, Ribela JS, Barbosa PF. Bundles de prevenção de pneumonia associada à ventilação mecânica: a importância da multidisciplinaridade. Perspec Online: Biol Saúde [Internet]. 2017 [acesso em I5 nov 20l8]; 7 (25):25-35. Disponível em: http://dx.doi. org/10.25242/886872520171200

22. Folco CP, Cruz ICF. Lung injury associated with mechanical ventilator: systematic literature review for a clinical protocol.J Spec Nurs Care. 2016; 8 (I): I- I I.

23. Derhun FM, Souza VS, Costa MAR, Inoue KC, Matsuda LM. Knowledge of nursing professionals regarding hand hygiene. Cogitare Enferm. 20 I6; 2 I (3): I-7.

24. Pulzi SA, Ferraz RRN, Lapchick MS. Pneumonia associated with mechanical ventilation as an indicator of health quality and safety. Rev Med Minas Gerais. 20I5; 25 (4):5 I7-522.

Recebido: 2019-10-| I

Aceito: 2019-11-12 